RELATO DE CASO

\title{
Síndrome de Morbihan: relato de caso e revisão bibliográfica
}

\section{Morbihan syndrome: case report and literature review}

\author{
Marina Macellaroํ․ Livia Matida Gontijo ${ }^{1}$. Renata Cristina Vasconcellos ${ }^{1}$. Nathalia Hatsue Oushiro ${ }^{1}$. Renan \\ Lage $^{2}$.
}

1 Residente de Dermatologia, Pontifícia Universidade Católica de Campinas, Campinas, São Paulo, Brasil. 2 Médico, Especialista em Dermatologia pela Sociedade Brasileira de Dermatologia. Assistente no ambulatório de Dermatologia, Pontifícia Universidade Católica de Campinas, Campinas, São Paulo, Brasil.

\section{RESUMO}

Síndrome de Morbihan é uma rara entidade, mais comum na terceira ou quarta década de vida. Afeta principalmente caucasianos adultos de ambos os sexos, apresentando incidência maior nas mulheres. É considerada uma forma de rosácea tendo como sinônimos linfedema rosáceo e edema facial persistente sólido. Sua patogênese não é completamente conhecida. Clinicamente caracterizada pelo aparecimento lento de eritema e edema sólido nos dois terços superiores da face, com acentuação na região periorbital, frontal, glabelar, nasal e bucinadora. Neste relato, apresentamos um caso de uma paciente do sexo feminino com linfedema de face, com hipótese diagnóstica inicial de Síndrome Linfocitária de Jessner. Após acompanhamento clínico e exame anatomopatológico confirmou-se o diagnóstico de Síndrome de Morbihan. O tratamento proposto foi doxiciclina em monoterapia com obtenção de sucesso terapêutico.

Palavras-chave: Linfedema. Rosácea. Terapêutica.

\section{ABSTRACT}

Morbihan Syndrome is a rare entity, most common in the third or fourth decade of life. It mainly affects Caucasian adults with higher incidence in women. It is considered a form of rosacea which is also referred to as rosaceous lymphedema and solid persistent facial edema. The underlying pathogenic mechanisms are unclear. Clinically characterized by persistent erythema and solid edema of the upper two-thirds of the face, particularly of the forehead, glabella, eyelids, nose and cheeks. In this report, we present a case of a female patient with face lymphedema, with an initial hypothesis of Jessner's Lymphocyte Syndrome. After clinical progression and anatomopathological examination the diagnosis of Morbihan syndrome was confirmed. We report a case of Morbihan Syndrome that were successfully treated with oral doxycycline therapy.

Keywords: Lymphedema. Rosacea. Therapy.

Autor correspondente: Marina Macellaro, Avenida John Boyd Dunlop, sem número, prédio dos ambulatórios, Jardim Ipaussurama, Campinas, São Paulo. CEP: 13034-685. Telefone: +55 19 3343-8600/19 99123-4949. E-mail: marinamacellaro@hotmail.com

Conflito de interesses: Não há qualquer conflito de interesses por parte de qualquer um dos autores.

Recebido em: 08 Jan 2018; Revisado em: 04 Abr 2018; Aceito em: 15 Mai 2018. 


\section{INTRODUÇÃO}

O termo doença de Morbihan é derivado da região francesa onde o primeiro paciente foi observado. ${ }^{1-3}$ Porém, a doença foi descrita pela primeira vez em 1957 por Robert Degos. ${ }^{4}$ Por apresentar diversos fatores etiopatogênicos, o termo "Síndrome de Morbihan" é mais aceito.

Segundo Veraldi et $\mathrm{al}^{2}{ }^{2}$ a Síndrome de Morbihan afeta principalmente caucasianos adultos de ambos sexos, porém, segundo a maioria dos autores é mais comum em mulheres. ${ }^{5,6}$

A patogênese da síndrome não é bem elucidada. Parece consenso entre os autores tratar-se de uma variante da acne ou da rosácea. Kou et $\mathrm{al}^{7}$ descreveram um caso de Síndrome de Morbihan associada ao Lúpus Extrafacial Miliar Disseminado e interrogaram se ambas manifestações dermatológicas apresentam mesmo mecanismo patogênico dentro do espectro da rosácea. Para outros, a síndrome pode ser causada por anormalidades nos vasos linfáticos, ${ }^{8}$ por obstrução ao fluxo linfático ou pela perda da integridade dos vasos linfáticos devido à destruição da elastina em torno das paredes vasculares pelo processo inflamatório crônico com consequente transudação de fluidos e linfedema. ${ }^{5,9,10}$ Wohlrab et al ${ }^{11}$ relataram alteração na drenagem linfática de pacientes com Síndrome de Morbihan através da ultrassonografia e da fluxometria laser Doppler. Suas descobertas sugerem a insuficiência vascular linfática como uma possível causa, porém, não há como verificar se essa alteração ocorreu posteriormente a um quadro inflamatório, como na rosácea, ou de forma isolada.

Clinicamente, a síndrome é caracterizada por eritema e edema sólido insidiosos da porção superior da face, com acentuação na região frontal, nasal, glabelar, bucinadora e palpebral. Telangiectasias, flushing facial, pápulas, pústulas, e nódulos podem estar presentes. ${ }^{12,13}$ As alterações cutâneas persistem indefinidamente sem tendência a involução espontânea. Alterações tendem a ser inicialmente flutuantes e posteriormente tornam-se permanentes, causando edema e distorção do contorno da face, ${ }^{14}$ podendo ocorrer subsequente estreitamento do campo visual. ${ }^{11,15}$ Blefarite e conjuntivite são as apresentações oculares mais comuns. ${ }^{16}$

Alterações laboratoriais são inespecíficas ou não encontradas, histopatologia e colorações específicas são importantes para afastar outras condições, como a metástase periorbitária, um importante diagnóstico diferencial. Outros diagnósticos diferenciais incluem granulomatose orofacial, sarcoidose, hanseníase, Lúpus Eritematoso Sistêmico (LES), leishmaniose cutânea, granuloma de corpo estranho, granuloma facial, síndrome da veia cava superior, escleredema de Buschke e Síndrome Linfocitária de Jessner, como no presente caso. ${ }^{5}$

Diversas opções de tratamento são sugeridas utilizando drogas sistêmicas geralmente com doses altas e por períodos prolongados. Porém, nem todo paciente responde ao tratamento proposto.

O objetivo deste estudo é demonstrar uma paciente com diagnóstico de Síndrome de Morbihan sem história prévia de rosácea, que apresentou resposta significativa com uso de doxiciclina oral.

\section{RELATO DE CASO}

Paciente do sexo feminino, 28 anos, procedente de Campinas, São Paulo, sem comorbidades prévias, procurou o ambulatório de Dermatologia apresentando eritema e edema endurecido nas regiões malares e na fronte (Figura 1). Apresentava cefaleia acompanhando o quadro cutâneo. Ao exame dermatológico apresentava placa eritematoedematosa, lisa e endurecida no terço superior da face. O exame anatomopatológico evidenciou infiltrado perivascular linfocitário e histiocitário de grandes células e células de tamanho intermediário com imunohistoquímica positiva para marcadores linfocitários de imunofenótipos $\mathrm{B}$ e $\mathrm{T}$, de padrão politípico reacional. No primeiro momento foi aventada hipótese de Infiltrado Linfocítico de Jessner e iniciado tratamento com hidroxicloroquina $400 \mathrm{mg}$ e prednisona $10 \mathrm{mg}$ empiricamente.

Figura 1. (A): Eritema e edema duro nas regiões malares e no fronte - visão frontal; (B): Eritema e edema duro nas regiões malares e no fronte - visão lateral esquerda; (C): Eritema e edema duro nas regiões malares e no fronte- visão lateral direita.
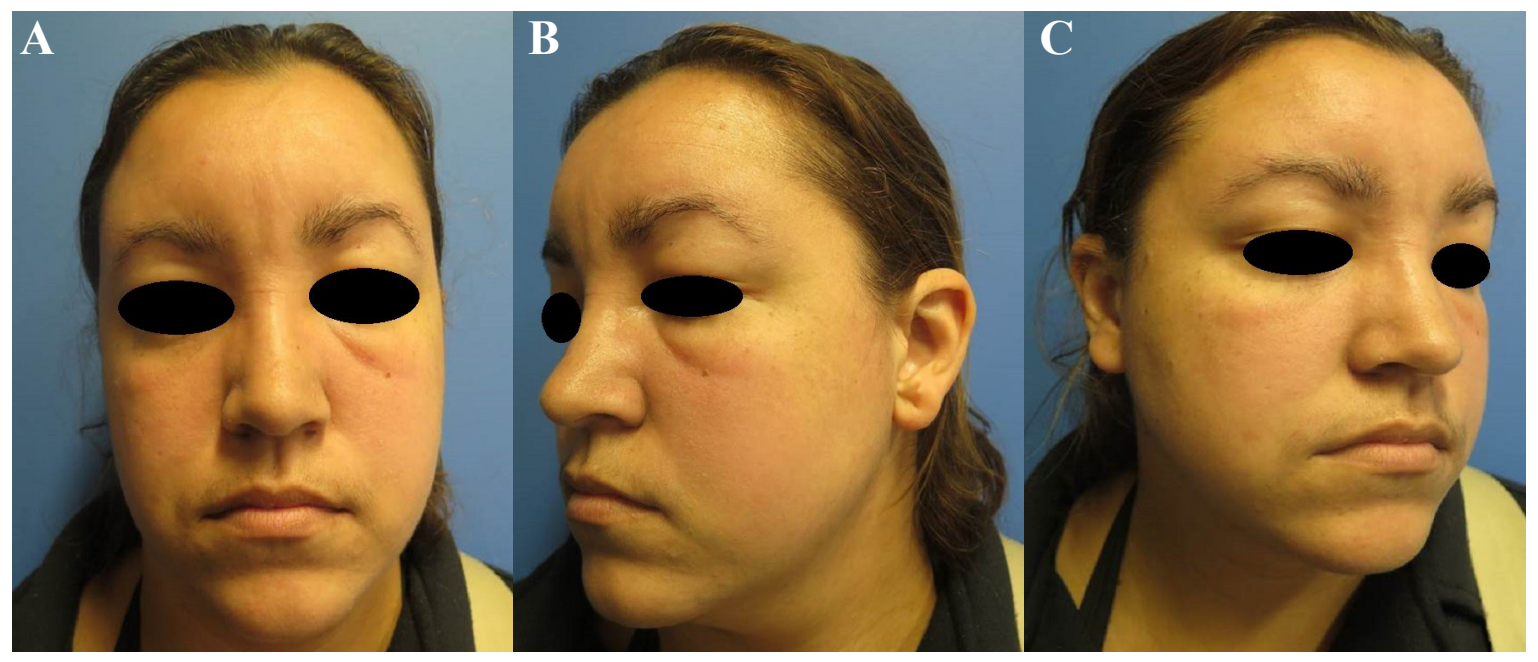
Devido a não melhora com o tratamento proposto e a evolução com flutuação do quadro clínico, foi optada pela realização de um novo exame histopatológico que evidenciou um infiltrado linfohistiocitário perivascular, por vezes atingindo o complexo pilossebáceo, além da presença de fibrose em diferentes áreas da derme adjacente ao componente inflamatório descrito. Os achados excluíram a hipótese de Síndrome Linfocitária de Jessner. Devido quadro clínico, evolução e anatomopatológico compatíveis foi confirmada a hipótese de Síndrome de Morbihan e introduzida doxiciclina $100 \mathrm{mg}$ de 12/12 horas com remissão completa do quadro clínico após 30 dias de tratamento (Figura 2).

Figura 2. (A): Remissão completa do quadro clínico após 30 dias de tratamento - visão frontal; (B): Remissão completa do quadro clínico após 30 dias de tratamento- visão lateral esquerda; (C): Remissão completa do quadro clínico após 30 dias de tratamento- visão lateral direita.
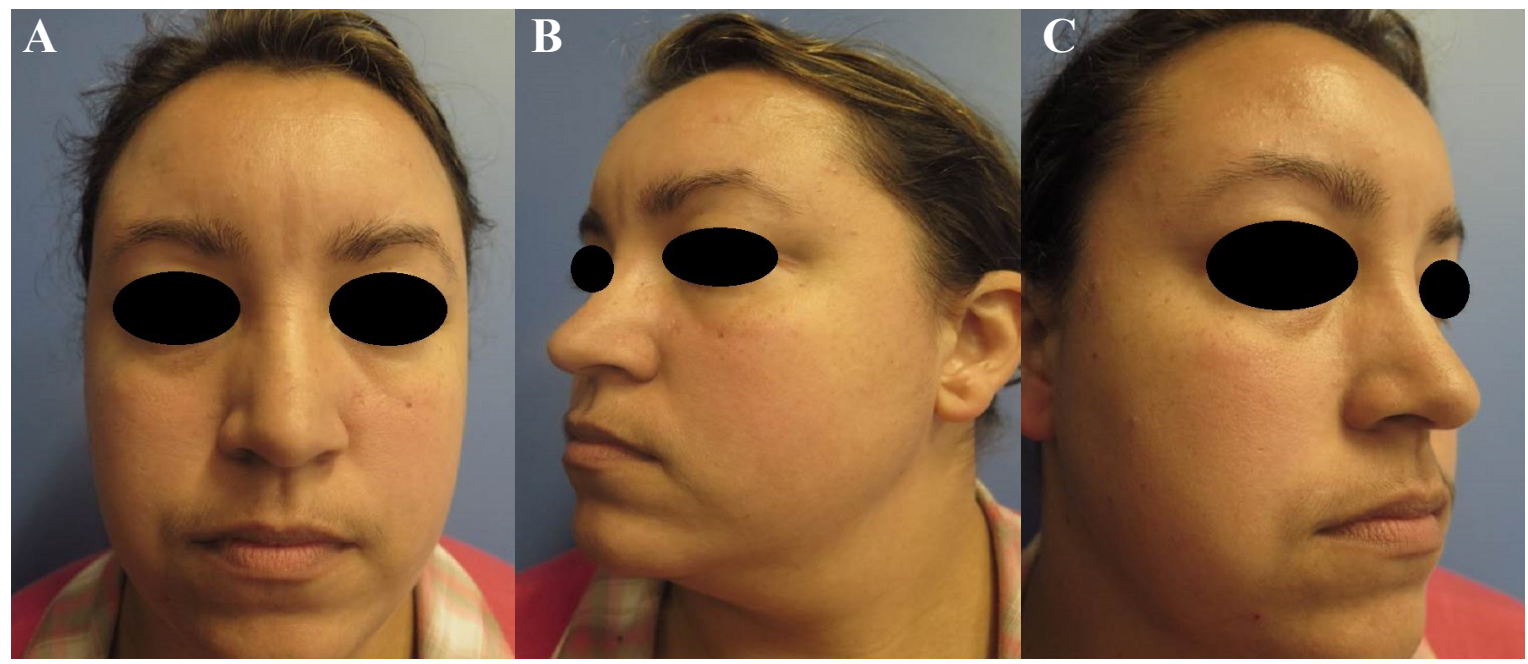

\section{DISCUSSÃO}

A Síndrome de Morbihan é caracterizada pelo desenvolvimento de edema endurecido principalmente nos dois terços superiores da face. A doença usualmente ocorre na terceira ou quarta décadas de vida. ${ }^{6}$ Nosso caso trata-se de uma mulher de 28 anos com diagnóstico clínico patológico sugestivo de Síndrome de Morbihan.

O anatomopatológico, embora inespecífico, é caracterizado por edema perivascular e infiltrado linfohistiocitário perianexial contendo numerosos mastócitos e dilatação dos vasos linfáticos. Granulomas linfohistiocitários com distribuição perianexial podem ser encontrados e hiperplasia da glândula sebácea pode ser observada em pacientes que tem ou tiveram rosácea. As características histopatológicas do linfedema persistente só foram documentadas em alguns relatos de casos, que incluem fibrose perifolicular e infiltrado perivascular e perifolicular de linfócitos, histiocitos e neutrófilos em terreno edematoso. ${ }^{16}$ Chaidemenos et $\mathrm{al}^{17}$ descreve ácaros Demodex nas aberturas foliculares.

O tratamento é desafiador e as evidências limitadas. Não há diretrizes para o tratamento da Síndrome de Morbihan. As terapêuticas mais comumente adotadas incluem uso de antibióticos e drenagem linfática da face. Várias drogas sistêmicas vêm sendo utilizadas incluindo talidomida, clofazimina, interferon gama, anti-histamínicos, prednisona, metronidazol, tetraciclinas, quinolonas como o fleroxacino ${ }^{18}$ e esteroides. ${ }^{14}$ No entanto, resultados mais promissores foram observados com a isotretinoína que foi inicialmente descrita por Jansen e Plewig em 1997. ${ }^{19}$ A dose da isotretinoína na literatura varia de 10 a $50 \mathrm{mg}^{19-22}$ ou de 0.1 a $0.2 \mathrm{mg} / \mathrm{kg} /$ dia $^{14}$ por 3-24 semanas ${ }^{5,19}$ em combinação com o cetotifeno (1-2 mg 12/12h) que interfere diretamente na degranulação dos mastócitos ${ }^{5}$ ou com a clofazimina. ${ }^{22,23}$ Porém, segundo a literatura, $15-20 \%$ dos pacientes que utilizam isotretinoína não apresentam boa resposta. ${ }^{2}$ Carruth et $\mathrm{al}^{24}$ relata terapia com agentes biológicos como uma terapêutica promissora futura. Exérese de tecido redundante pode ser uma alternativa e um grupo de pacientes relatou bons resultados cosméticos com blefaroplastia por laser de $\mathrm{CO}_{2} \cdot{ }^{14}$

Em 2015 Fujimoto et $\mathrm{al}^{23}$ descreveram o primeiro caso da literatura inglesa de Síndrome de Morbihan tratado com tetraciclina e a partir de então outros estudos também evidenciaram bons resultados com esta classe de antibióticos. No caso relatado por Fujimoto, ${ }^{23}$ inicialmente administrou-se betametasona oral por uma semana com agravamento do quadro clínico sendo então suspenso o corticoide e introduzido minociclina $50 \mathrm{mg} /$ dia por 4 semanas. Devido melhora apenas parcial a dosagem da minociclina foi dobrada, com desaparecimento do edema e do eritema faciais após 4 semanas de tratamento. Não houve recidiva do quadro nos 8 meses seguintes ao tratamento. Neste artigo especula-se que o uso prolongado da isotretinoína oral ou tetraciclinas seja eficaz apenas nos casos de Síndrome de Morbihan com infiltração abundante de mastócitos. Okubo et a ${ }^{25}$ concluíram que mais pesquisas são necessárias para compreender o mecanismo de ação das tetraciclinas nesta síndrome. 
Chaidemenos et a ${ }^{17}$ utilizaram doxiciclina $200 \mathrm{mg} / \mathrm{dia}$ por dois meses com melhora clínica do paciente, com posterior dose de manutenção por 6 meses com doxiciclina $40 \mathrm{mg} /$ dia. Após 51 meses da finalização do tratamento, o paciente mantinha remissão completa do quadro.

Ranu et al ${ }^{16}$ utilizaram doxiciclina $100 \mathrm{mg}$ 12/12h durante 6 semanas com melhora do eritema, porém, com persistência do edema. Devido queixa de visão turva optou-se pela adição de prednisolona oral $20 \mathrm{mg} / \mathrm{dia}$. Após o uso de prednisolona $20 \mathrm{mg}$ por 2 semanas com posterior desmame para $10 \mathrm{mg}$ por mais 1 semana, o edema e o eritema diminuíram notavelmente. Após 2 semanas de uso da prednisolona e 12 semanas de uso da doxiciclina, o paciente apresentou melhora completa do quadro clínico.

Okubo et $\mathrm{al}^{25}$ descreveram quatro casos clínicos de Síndrome de Morbihan. Em dois casos iniciaram-se prednisolona oral e corticoide intralesional sem sucesso. Em um paciente foi iniciada minociclina, porém, devido a disfunção hepática,

\section{REFERÊNCIAS}

1. Nagasaka T, Koyama T, Matsumura K, Chen KR. Persistent lymphoedema in morbihan disease: formation of perilymphatic epithelioid cell granulomas as a possible pathogenesis. Clin Exp Dermatol. 2008;33(6):764-7.

2. Veraldi S, Persico MC, Francia C. Morbihan syndrome. Indian Dermatol Online J. 2013;4(2):122-4.

3. Degos R, Civatte J, Beuve-Méry M. Nouveau cas d'oedème érythémateux facial chronique. Bull Soc Fr Dermatol Syph. 1973;80:257.

4. Gorin I, Gaitz JP, Chevrier C, Lessana-Leibowitch M, Fortier P, Escande JP. Maladie du morbihan: essais therapeutiques. Deuxième présentation. Journées Dermatologiques de Paris. 1991;111.

5. Vasconcelos RC, Eid NT, Eid RT, Moriya FS, Braga BB, Michalany AO. Morbihan syndrome: a case report and literature review. An Bras Dermatol. 2016;91(5 Supl 1):157-9.

6. Marks R, Rook A, Ebling FJ, Wilkinson DS, Champion RH. Rosacea flushing and perioral dermatites. Rook's textbook of dermatology. 5. ed. Londres: Blackwell Scientific Publications; 1992.

7. Kou K, Chin K, Matsukura S, Sasaki T, Nozawa A, Aihara M, et al. Morbihan disease and extrafacial lupus miliaris disseminatus faciei: a case report. Ann Saudi Med. 2014;34(4):351-3.

8. Jungfer B, Jansen T, Przybilla B, Plewig G. Solid persistent facial edema of acne: Successful treatment with isotretinoin and ketotifen. Dermatology. 1993;187(1):34-7.

9. Rebora A. Rosacea. J Invest Dermatol. 1987;88(3 suppl):56s-60s.

10. Wilkin JK. Rosacea. Pathophysiology and treatment. Arch Dermatol. 1994;130(3):359-62.

11. Wohlrab J, Lueftl M, Marsch WC. Persistent erythema and edema of the midthird and upper aspect of the face (morbus morbihan): optou-se pela mudança para doxiciclina pelo seu perfil mais brando de toxicidade hepática e renal e superioridade farmacocinética em relação à absorção da droga. Os quatro casos tiveram bons resultados com doxiciclina $200 \mathrm{mg} /$ dia. A duração dos tratamentos variou de 5 a 12 meses e nenhum dos casos apresentou recorrência após descontinuação da medicação.

Segundo Carney et al, ${ }^{26}$ o uso de corticosteroides sistêmicos não foi satisfatório: a prednisolona $50 \mathrm{mg} / \mathrm{dia}$ por um mês foi ineficaz, prednisolona $20 \mathrm{mg} /$ dia (duração não disponível) mostrou apenas efeito transitório e um curso curto de corticosteroide não forneceu nenhuma melhoria.

No nosso caso optamos pela monoterapia de tetraciclina ao invés da associação com corticoide. A doxiciclina foi escolhida no nosso caso em detrimento de outras tetraciclinas devido facilidade de acesso à droga na Unidade Básica de Saúde do Sistema Único de Saúde brasileiro. Paciente permanece com doxiciclina em baixas doses para estabilidade do quadro cutâneo.

evidence of hidden immunologic contact urticaria and impaired lymphatic drainage. J Am Acad Dermatol. 2005;52(4):595-602.

12. Scerri L, Saihan EM. Persistent facial swelling in a patient with rosacea. Rosacea lymphedema. Arch Dermatol. 1995;131(9):1071-4.

13. Reinholz M, Tietze JK, Kilian K, Schaller M, Schöfer H, Lehmann P, et al. Rosacea - S1 guideline. J Dtsch Dermatol Ges. 2013;11:768-80.

14. Hu SW, Robinson M, Meehan SA, Cohen DE. Morbihan disease. Dermatol Online J. 2012;18(12):27.

15. Morales-Burgos A, Alvarez Del Manzano G, Sánchez JL, Cruz CL. Persistent eyelid swelling in a patient with rosacea. P R Health Sci J. 2009;28(1):80-2.

16. Ranu H, Lee J, Hee TH. Therapeutic hotline: successful treatment of morbihan's disease with oral prednisolone and doxycycline. Dermatol Ther. 2010;23(6):682-5.

17. Chaidemenos G, Apalla Z. Sidiropoulos. Morbihan disease: successful treatment with slow-releasing doxycycline monohydrate. J Eur Acad Dermatol Venereol. 2018;32(2):e68-9.

18. Uhara H, Kawachi S, Saida T. Solid facial edema in a patient with rosacea. J Dermatol. 2000;27(3):214-6.

19. Smith LA, Cohen DE. Successful long-term use of oral isotretinoin for the management of Morbihan disease: a case series report and review of the literature. Arch Dermatol. 2012;148(12):1395-8.

20. Batra M, Bansal C, Tulsyan S. Granulomatous rosacea: unusual presentation as solitary plaque. Dermatol Online J. 2011;17(2):9.

21. Mazzatenta C, Giorgino G, Rubegni P, de Aloe G, Fimiani M. Solid persistent facial oedema (Morbihan's disease) following rosacea, successfully treated with isotretinoin and ketotifen. Br J Dermatol. 1997;137(6):1020-1. 
22. Jansen T, Plewig G. The treatment of rosaceous lymphoedema. Clin Exp Dermatol. 1997;22(1):57.

23. Fujimoto N, Mitsuru M, Tanaka T. Successful treatment of morbihan disease with long-term minocycline and its association with mast cell infiltration. Acta Derm Venereol. 2015;95(3):368-9.

24. Carruth BP, Meyer DR, Wladis EJ, Bradley EA, Al-Rohil R, Jones DM, et al. Extreme eyelid lymphedema associated with rosacea (morbihan disease): case series, literature review, and therapeutic considerations. Ophthalmic Plast Reconstr Surg. 2017;33(3S Suppl 1):S34-8.

25. Okubo A, Takahashi K, Akasaka T. Four cases of morbihan disease successfully treated with doxycycline. J Dermatol. 2017;44(6):713-6.

26. Carney JW. Solid edema of face (??). Arch Dermatol. 1966;94(5):664-6.

\section{Como citar:}

Macellaro M, Gontijo LM, Vasconcellos RC, Oushiro NH, Lage R. Síndrome de Morbihan: relato de caso e revisão bibliográfica. Rev Med UFC. 2018 out-dez;58(4):61-65. 